\title{
Measurement and recording errors of the respiratory rate
}

\author{
Authors: Yang Chen, Josh Patch, Katy Rose, Wai Soon, Kate West, Myura Nagendran \\ and Denesh Srikantharajah
}

\begin{abstract}
Aims
To identify errors at the measurement and/or recording phase of the respiratory rate in the National Early Warning Score (NEWS).

\section{Methods}

During a 1-week period at three London hospitals, foundation year one (FY1) doctors manually counted and recorded the respiratory rate $(\mathrm{RR})$ into the medical notes during a ward round (WR). This was compared to what had been documented in the NEWS observation chart.

Three outcomes were measured: $1 \mathrm{a}$ - presence of a significant ( $>5 \mathrm{RR}$ ) discrepancy between documentation in NEWS chart and what the RR was on the WR; $1 \mathrm{~b}$ - presence of a specific RR value documented rather than ' $12-20$ '; $1 \mathrm{c}$ - presence of there being $>4$ hours since the WR and the last RR recorded; 2 - any change in behaviour of the medical team as a result of being told an accurate RR; 3 - number of times the FY1 was unable to measure the RR (for 30 seconds) because of the brevity of the interaction.
\end{abstract}

\section{Results}

Sample size: $\mathrm{N}=208 ; 1 \mathrm{a}-12$ instances $(5.8 \%)$ where there was a significant discrepancy (mean difference of nine); $1 \mathrm{~b}-39$ instances $(18.8 \%)$ where specific RR was documented; $1 \mathrm{~b}-23$ instances $(11.1 \%)$ where there was $>4$ hours since last observation; $2-$ One instance $(0.5 \%)$ of change of behavior; $3-49$ instances $(24.1 \%)$ where there was insufficient time to record RR.

\section{Conclusions}

We identified significant variation in the way that the RR was measured and documented in the NEWS at three London hospitals, all belonging to the same trust. We have arranged additional training for relevant stakeholders (nursing staff, healthcare assistants and other clinical staff) to ensure that this most sensitive of markers for illness becomes more accurately measured and recorded.

\section{Conflict of interest statement}

None declared. 